\title{
Mobile Networks and Internet of Things Infrastructures to Characterize Smart Human Mobility
}

\author{
Luís Rosa ${ }^{1, *(\mathbb{D})}$, Fábio Silva ${ }^{1,2} \mathbb{D}$ and Cesar Analide ${ }^{1}(\mathbb{D}$ \\ 1 ALGORITMI Centre, Department of Informatics, University of Minho, 4710-057 Braga, Portugal; \\ fas@estg.ipp.pt (F.S.); analide@di.uminho.pt (C.A.) \\ 2 CIICESI, School of Management and Technology, Politécnico do Porto, 4610-156 Felgueiras, Portugal \\ * Correspondence: id8123@alunos.uminho.pt
}

Citation: Rosa, L.; Silva, F.; Analide, C. Mobile Networks and Internet of Things Infrastructures to Characterize Smart Human Mobility. Smart Cities 2021, 4, 894-918. https://doi.org/ 10.3390/smartcities4020046

Academic Editor: Fernando De la Prieta

Received: 7 May 2021

Accepted: 7 June 2021

Published: 10 June 2021

Publisher's Note: MDPI stays neutral with regard to jurisdictional claims in published maps and institutional affiliations.

Copyright: (c) 2021 by the authors. Licensee MDPI, Basel, Switzerland. This article is an open access article distributed under the terms and conditions of the Creative Commons Attribution (CC BY) license (https:/ / creativecommons.org/licenses/by/ $4.0 /)$.

\begin{abstract}
The evolution of Mobile Networks and Internet of Things (IoT) architectures allows one to rethink the way smart cities infrastructures are designed and managed, and solve a number of problems in terms of human mobility. The territories that adopt the sensoring era can take advantage of this disruptive technology to improve the quality of mobility of their citizens and the rationalization of their resources. However, with this rapid development of smart terminals and infrastructures, as well as the proliferation of diversified applications, even current networks may not be able to completely meet quickly rising human mobility demands. Thus, they are facing many challenges and to cope with these challenges, different standards and projects have been proposed so far. Accordingly, Artificial Intelligence (AI) has been utilized as a new paradigm for the design and optimization of mobile networks with a high level of intelligence. The objective of this work is to identify and discuss the challenges of mobile networks, alongside IoT and AI, to characterize smart human mobility and to discuss some workable solutions to these challenges. Finally, based on this discussion, we propose paths for future smart human mobility researches.
\end{abstract}

Keywords: internet of things; mobile networks; smart human mobility; smart cities

\section{Introduction}

In the rise of the digital age, technology is placed at the heart of our society, from working to human mobility. The digital world impacts society positively, creating new trends in technology. Following these trends, the important step is an ecosystem of services and smart applications based on the interconnectivity of heterogeneous devices, namely, the use of cyber-physical environments (CPE) in the fusion of the physical worlds and digital. In other words, the contributions of Artificial Intelligence (AI), Internet of Things (IoT), and new network technologies has been accelerated the digital transformation of society [1].

Based on the growing digitalization of society, the increased density of IoT has been a natural phenomenon. In fact, the real value of IoT comes from the information provided by available devices and sensors. Information fusion improves estimation about the state of things and information quality and completeness. Consequently, these activities of the information fusion process lead to an increase in the number of connected users due to the trust in this process and the performance and the capacity of IoT smart applications. Therefore, so that this huge demand for interconnected devices is not compromised in terms of performance, simultaneous connection and response time to user requests, reducing latency to the final users and the deployed sensors, a set of requirements should be considered like spectral efficiency, signaling efficiency, great data rate and security. This means that a new wireless communication needs to be implemented to answer this migration. In other words, the next mobile generation technology, such as 5G, should address many challenges for better applications and connectivity of mobile users [2]. 
The growth mobile generation technologies, including wireless communications, has increased over recent decades [3]. In the past 50 years, each one of them has had an impact on "mobile society" or IoT device users. For example, $1 \mathrm{G}$ came when salespersons had a phone in their car so they could call orders while they were travelling. In $2 \mathrm{G}$, we were able to start sending text messages, via the short message service (SMS), to each other and enabled phones into people's pockets. In its turn, 3G phones were not limited to a certain region and began working everywhere. Inter-operability between various networks was offered using 4G. Lastly, 5G comes to increase coverage and to suit users [4].

With the development of ubiquitous wireless mobile networks and systems, different performance requirements of smart city services, such as human mobility, can be met due to flexibility of network. No matter where we are or what we are trying to achieve, a high speed, reliable, and robust wireless network is the first step to build a human mobility service. This is the backbone of all potential smart human mobility applications. For example, these applications include geo-tagged posts from social networks [5]; tracks from mobile phone GPS [6]; and communication records between cellular network and phones [7]. This amount of digital data fostered several scientific productions on human mobility behavior, such as trajectory data mining [8], and the uncovering of statistical patterns [9]. Additionally, the data of these applications provided relevant metrics about population mobility, i.e., when there are peaks in the number of people in the same place or moving less (or more) than usual. These metrics open up a new dimension for understanding human mobility behaviors.

Although ubiquitous, mobile phone data have their biases and limitations. Ordinarily, data stream of a semi-urban or urban population might be more representative than those living in rural areas. Popular analytical reports from large data providers show physical distancing (mobility) metrics, but the underlying data they represent and the aggregation methods used are typically not readily available. This scant transparency makes it hard to know the representativeness and limitations of these data before using it for modeling. A common framework is needed to analyze the characteristics of this disparate data and their outputs, to allow for better comparison across human mobility metrics and easier interpretation. On the other hand, data availability also enables human mobility forecasting tasks, i.e, next-location prediction, crowd flow prediction, and trajectory generation [10].

Currently, the literature is promoting towards the design of deep learning (DL) solutions $[11,12]$. They can deal more efficiently with human mobility forecasting tasks. Furthermore, they can extract relevant features from the heterogeneous and big data sources, facilitating the combination of contextual information (census data and weather data) and mobility data (trajectories and flows). On the other hand, thanks to a large number of recurrent neural network (RNN) algorithms, DL can capture non-linear spatio-temporal and sequential relationships in the data. Although this work focuses on employing DL, it also includes other prevalent prediction. For instance, adopt gravity models and spatial equilibrium models to predict flow changes methods.

One of the advantages of smart cities is that no matter how much technology is developed and enhanced, there is always chance to improve and reach a level that has not yet been reached. As we have seen, IoT has an impact on society, making it hyperconnected; mobile technology is more present than ever, anytime and anywhere, the wireless network reaches a considerable area and AI is increasingly a partner of prediction and guidance to the human. However, we have seen that all of them present their limitations and challenges in terms of the development of human mobility services. Although many studies have addressed and investigated the extent to which these technologies can be useful in human mobility, many of them address issues in an isolated way. Thus, this extensive article intends to bring together these different approaches, understand where we are at the level of implementation of these technologies in the analysis of mobility behaviors of individuals and, from them, indicate paths that can innovate human mobility services.

In this article, we discuss how important IoT, AI, and wireless network technologies are to the success of smart human mobility applications. Thus, we study the fundamental 
requirements of smart human mobility and leverage opportunities generated by the constant evolution of mobile connectivity and IoT architecture. Moreover, this study intends to survey the level of coverage of emerging technologies, IoT architectures and cellular networks technologies in human activity tracking. Finally, to present new smart human mobility solutions, we discuss future directions towards new mobile technologies.

\section{Fundamental Requirements of Smart Human Mobility}

Information and Communication Technology (ICT) predicts a growth in the number of devices that can communicate with each other, altering the perceived physical world into an information and knowledge system. This evolution has led to the development of Cloud and network/communication technologies and drives the evolution of IoT [13]. A broad range of research fields is benefiting from the data explosion caused by the IoT, including tracking, better insights into a range of real-world problems, as well as the ability to make critical operational decisions. Among them are those that research human mobility using machine learning (ML) algorithms and metrics. Therefore, it should be considered that we speak not only of human mobility research but of smart human mobility research.

\subsection{Ubiquitous Networking for the Internet of Things}

As the demand for mobile services increases, so do mobile wireless networks need to increase their capacities and density of their networks especially to support IoT devices. However, this also presents new technical challenges related to the increasing density of wireless communications and networks. To meet this growing demand, we need to select appropriate methods to overcome these limitations, which human mobility surveys that track people do not call into question.

Limitations on the availability of internet access in remote areas contrast with the clear abundance of pervasive broadband internet access in urban areas. This creates what can be called a digital divide, that disrupts the collection of data, which can be used for human mobility studies. The major problem is that the cost of operating such high availability internet services in these areas is rarely economically sustainable. Figure 1 provides a high-level approximation of wireless coverage in Europe.

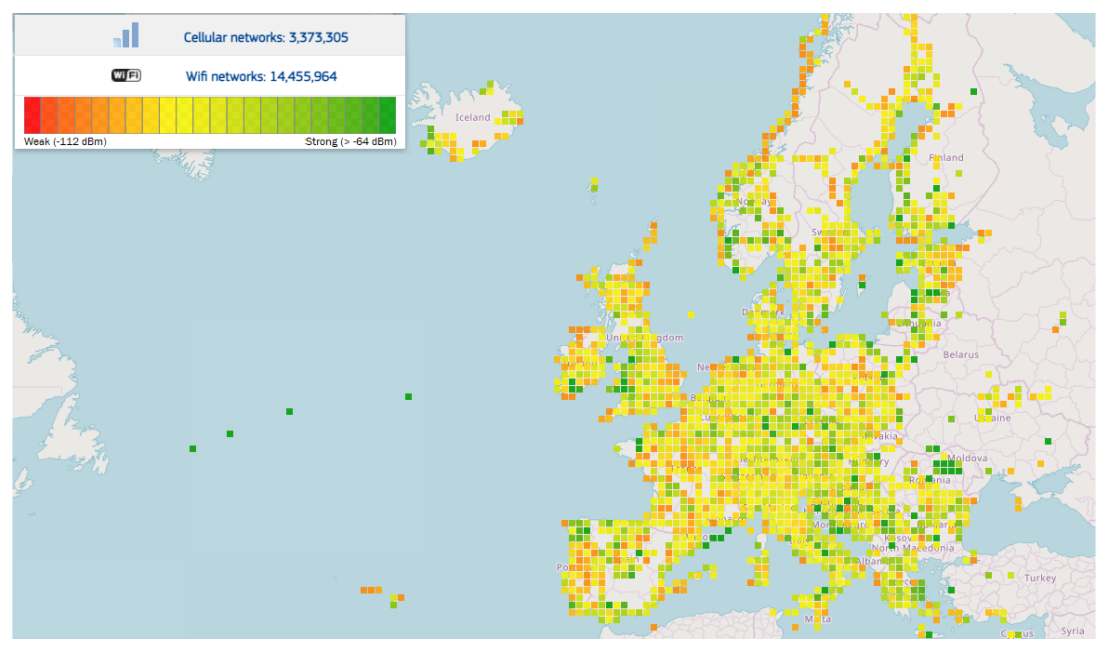

Figure 1. Areas covered by Cellular and WiFi networks (based on [14]).

There are gaps in coverage that are shown by this high-level approximation. Nevertheless, actual coverage may differ from the above map and may be affected by terrain, weather, network changes, buildings, construction work, signal strength, high-usage periods, and other factors. To overcome these challenges, new broadband wireless access technologies to operate in both urban and rural areas are required. These technologies should address important use cases, including applications for human mobility tracking or human mobility forecast tasks. However, recent research has explored this subject, 
and there are several fundamental and technological challenges that should be considered in urban or remote and rural scenarios.

\subsubsection{Urban Scenario}

Smart human mobility projects have been among the most actively discussed realizations, which are made possible by a purposeful and integrated IoT, data, and connectivity approach, leveraging a mix of challenges and solutions in urban scenarios.

Data collection in smart cities is a challenge for researchers and authorities. Since the transformation of data into information raises privacy issues, there are several barriers that should be overcome in human mobility surveys. The data generated in these environments is often large, and special methods to handle such large streams are required. Big data is a term coined to gather information technology that enables this. It provides means to handle data streams, data collection, data transformation, and information generation from data streams. Categorizing different use cases for big data and smart cities may present technological advantages in the planning of new $R \& D$ projects targeting high volume data streams and smart city applications [15].

In Figure 2, the category of local preventive management depends on individual customer data, including transport cards, call location records, and individual messages from your service provider. The objective is to understand the root causes of citizens' mobility problems based on data and to anticipate solutions, which increases the efficiency of administration and avoids resource waste. For example, the dynamics of human activity recorded in [16] was used to predict crimes. The category for local operational management is based on data from local service providers, such as urban infrastructure and company resource data. The objective is to improve the operational processes of local governments and companies based on improving communication between resources, infrastructure, and citizens or visitors using data. Understanding human mobility and urban dynamics is vital for public transport operators, urban planners, and location-based service providers [17]. The category of local network development depends on user data to help them. This category improves the accessibility of user resource local networks based on a better understanding of these users. For example, while most existing empirical human mobility studies are based on location data from cell phones, [18] uses user's call history to record users' locations when they receive or initiate a text message or call. Finally, the 'local information diffusion' category depends on data from local service providers to help users. It has the goal to increase efficiency and identify useful information regarding cities, citizens and its visitors. This effort is often governed by data policies such as open data, where data are freely available to everyone. For instance, mobility data in [19] from Descartes Labs is available free of charge at the United States county level.

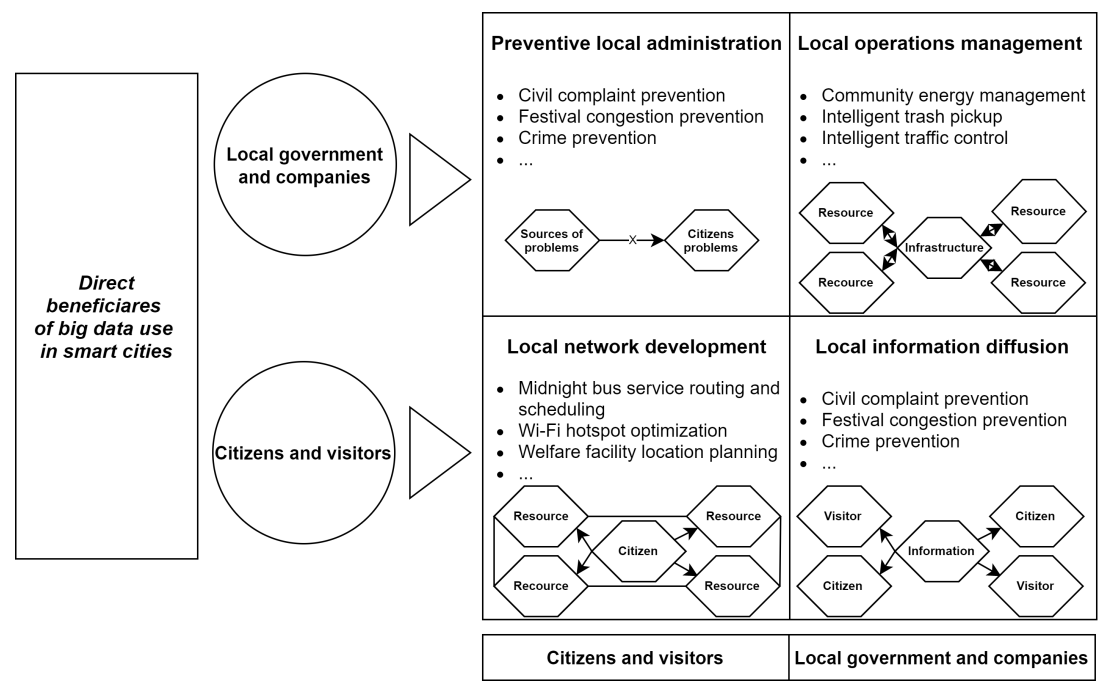

Figure 2. Categorization of use cases for big data and smart cities. 
Another challenge comes into view regarding data handling, data fusion, and information creation in the context of smart cities. From the collection of data to the delivery of added value information in smart cities, challenges can be identified, as illustrated in Figure 3.

For starters, data quality management is a prerequisite for trustworthy smart city information. This concerns aspects such as data isolation, data unavailability, data quality, and data costs [20]. Processes of data and information fusion with the objective to integrate different streams or sources of data inside smart cities or IoT applications is another challenge that can be identified.

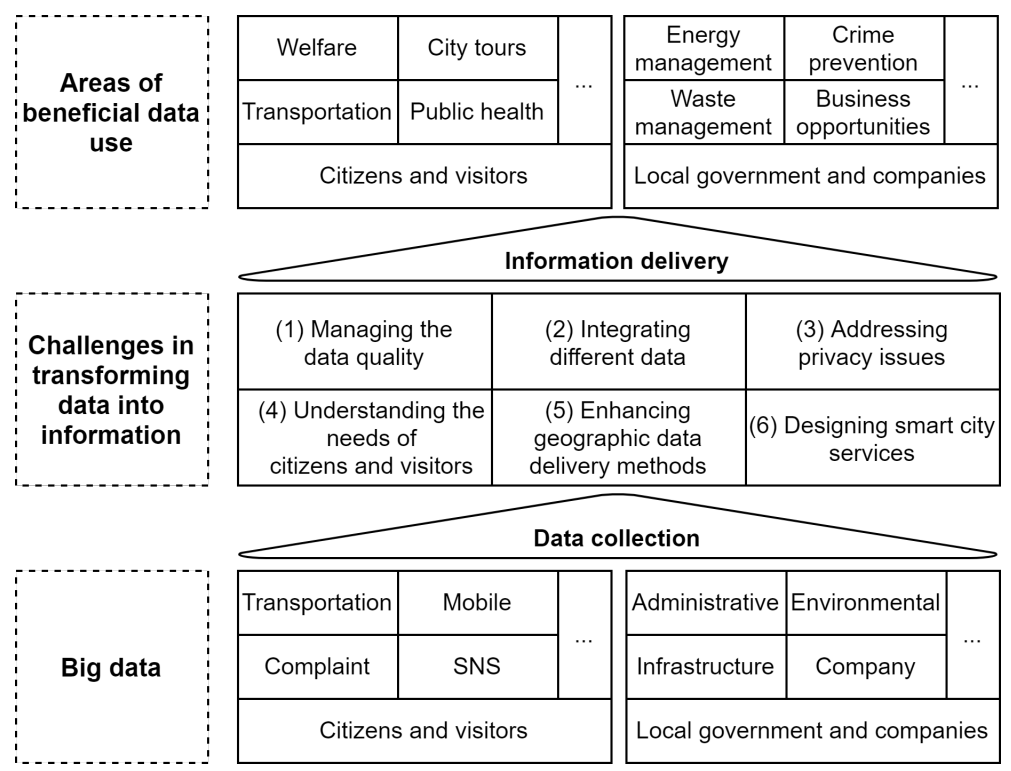

Figure 3. Challenges in transforming data into information for smart cities.

Figure 3 demonstrated different categories of data that can be collected from different multiple sources to create higher quality information for the betterment of citizens' lives. Nevertheless, linking data from different sources often comes with its own challenges due to organizational nature, data structures or interpretation. To address this problem in [21], an architecture, $m P a t$, is proposed to explore issues related with multi-source data applied to the specific case of human mobility.

The third challenge is data privacy. In project [22], authors analyzed over 580 million tweets worldwide to see how global collaborative efforts in reducing human mobility are reflected from the user-generated information at the global, country, and USA state scale. Nevertheless, social media data are relatively less privacy-concerning compared to passive data-collecting approaches that include phone calls, cellular records, and smart cards. Researchers applied methods that identified and aggregated before application. Then, understanding the needs of citizens, visitors, and employees is another challenge. An expert interviewed in [23] highlighted that the design and development of platforms for big data processing should be a priority, with a focus on citizen-oriented management. In terms of geographic information delivery methods, data should be analyzed according to a geographic unit (e.g., region and building). For example, [24] proposes a series of standard modifications to the models, such as using travel time instead of physical distance and adding a multiplicative term to correct the underestimated urban-to-urban trip volumes. Finally, designing a data-based smart city service is crucial because this task integrates all of the outcomes from the data analytics, ideation, and information content design for a smart city [25]. 


\subsubsection{Remote or Rural Scenario}

Concepts such as Smart Cities and IoT are often used together, especially when talking about next gen cities and city innovation. IoT is viewed as the underlying infrastructure that enables the realization of a Smart City. Although urban areas are often a prime example of the adoption of new and innovative technologies, rural and remote cities cannot be disregarded from this new innovation cycle. There are, in fact, use cases and needs for Smart City technology applied to remote and rural areas, and more importantly, adapted for such reality. An IoT and mobile networks framework should be developed according to rural needs. In the case of human mobility, it should be employed to assess human mobility innovations that answer the needs of the different rural contexts [26]. However, rural mobility also presents many challenges relevant to IoT application. First, remote areas tend to lack communication network infrastructure. Second, local authorities should be offered the right incentives to buy into IoT systems, whose upfront installation costs are still quite expensive. The good news is that there are entities and initiatives that have already begun to tackle these challenges. For example, [27] uses low-consumption and long-range networks called long-range (LoRa) communications to remotely monitor the health conditions and living standards of elder people, spread over large rural areas with limited to no mobile network coverage. Another example can be found in [28], where communities live in the Peru Amazon rainforest without access to communication services. In this work, a model that is based on the notion of small rural operator (SRO) is presented. The SRO directly targets the use case of communication needs of isolated communities in rural areas. In [29], the Sigfox network is used to collect messages obtained between the cities of Antwerp and Ghent granting network coverage complementing standard communication infrastructure. Figure 4 presents a map with Sigfox transmission locations.

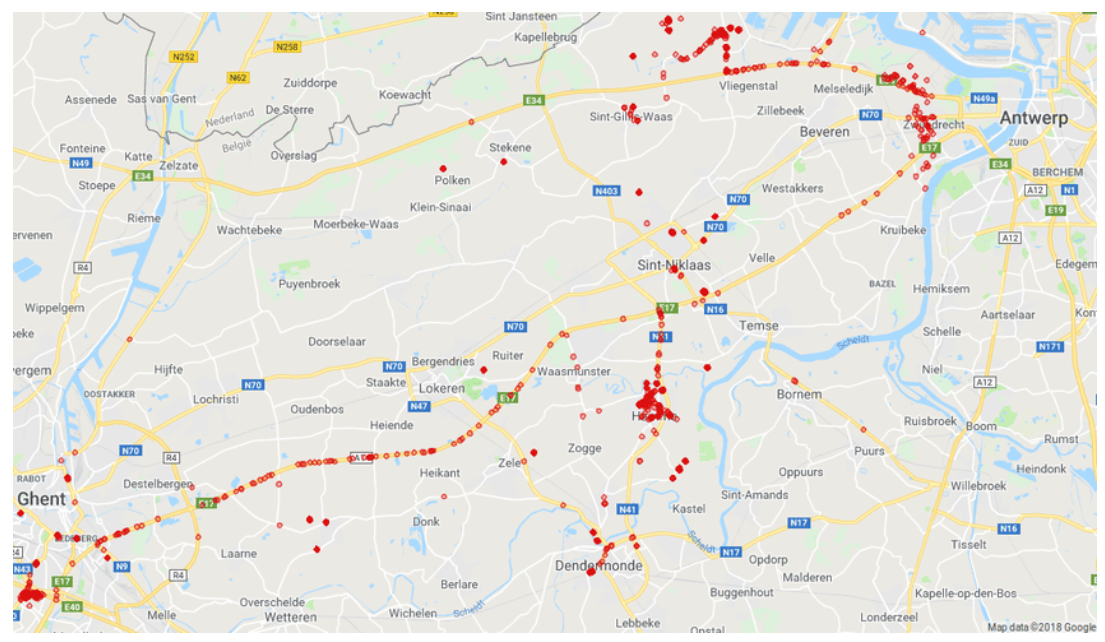

Figure 4. Sigfox transmission locations are indicated by red dots on the map (based on [29]).

Another typical difficulty associated with using IoT and mobile networks in rural areas is security. The possibility of data breaches should be addressed before using IoT. However, solutions like Bridgera IoT [30], a custom cloud platform, provides individuals with a sense of security where the capability to locate and track people and assets in real-time is made possible with IoT technology. Additionally, there are a lot of devices with proprietary technology in the IoT which makes horizontal communication between devices hard. Lack of interoperability with increasing costs is the biggest barrier to the use of IoT in rural areas. To counter this trend, the European Union (EU) is committed to sponsoring, new developments and research and innovation (R\&D) targeting rural digital ecosystems and service platforms, through a $€ 30$ million initiative and a new term, "smart village" for focusing the approach to rural and remote areas [31]. The two new large-scale pilot projects ("OrganiCity: Co-creating smart cities of the future" and "IoF2020: Internet of Food \& Farm 2020") represent an example of how interoperability mechanisms can 
be used to link to smart villages in remote areas. Ultimately, although data protection, privacy, and cross-border data exchange are important features that can be violated with the extreme connectivity of IoT, some projects have been trying to find solutions for this challenge. One of them uses five characteristics of the diffusion of innovation (DOI) theory to explain the organization's IoT security strategy. The study [32] suggested that the five characteristics of the DOI theory and the awareness and security of the external factors were critical in the factors that influenced the adoption of internet banking in South African rural areas. Findings reveal that the intent to adopt internet banking services could be predicted by variables directly linked to the existence of capable communications networks. Such variables included awareness, compatibility, trialability, and security.

\subsection{Metrics in Human Mobility Data Network}

With the technology currently available, it is possible to combine devices with heterogeneous systems, such as smartphones with mobile networks (3G/4G and, in the future, $5 \mathrm{G})$, Bluetooth devices, wireless networks, sensors, among others, to disseminate human movement data and its analysis. These data holds an immense promise for understanding human behavior on a vast scale, and with a precision and accuracy never before possible with censuses, surveys, or other existing data collection techniques. However, it is needed to discuss challenges with existing data sources and describe metrics for measuring human mobility.

\subsubsection{Human Mobility Data Sources}

Thanks to new network technologies, finding a dataset to analyze people's mobility is not a big concern any more. However, an effort should be made to overcome the challenges to lead with different types of datasets, including phone call detail records (CDR), wi-fi or radio-frequency identification (RFID), location-based social network (LBSN) data, and global positioning systems (GPS), in order to obtain useful information from geographical movements.

CDRs are collected by mobile network operators in the course of providing their service and do not require the use of smartphones. Therefore, their use in research does not preclude those from low socio-economic groups in the way that GPS data would, for instance. This is also valuable for countries where there are no integrated infrastructures for population census [33]. However, there are limitations that need to be taken into account in evaluating the use of CDRs in human mobility research. For studies that require CDRs to be collected by multiple operators, data availability, formats, and levels of aggregation vary, and this will influence the type of analysis that can be done [34]. There is an unknown level of discrepancy between phone owner and main user when phones are shared or bought for another person. This can call into question the validity of study findings focusing on particular demographics, since the extent of group representation is not known. In this work, CDR dataset is associated with a phone that made a voice call with the locations (latitude and longitude) of the cellular towers and reconstructs the spatio-temporal data of a mobile phone. Our research use 311 calls or service requests in New York City from January 2011 to December 2018 [35].

Wi-fi or RFID raises privacy issues since it collects involuntary information about devices, that can be used to infer information about their users using the MAC addresses and the service set identifiers (SSID) broadcasted. In addition, data collected can often reveal the last wi-fi spots where the device was connected. Therefore, new challenges should be dealt with, regarding the methods for analyzing the captured information which affects the methods used for unique device counts. Nevertheless, these measures aim to prevent the passive tracking and involuntary information capturing. To solve this problem, the current operating systems tend to broadcast less SSIDs, and that tactic will be more popular in the future where the only SSIDs broadcasted will be ones from hidden networks. This is done because the access points require this information to be broadcasted, in order to respond to the probe requests, thus still leaving an opportunity to analyze SSID 
information [36]. In the case of wi-fi, we use the historical listing of LinkNYC Kiosks that save their location, and the status of the Link's wi-fi, tablet, latitude, longitude, geometry point, type of kiosk, registry date, and phone [37]. These devices are connected daily for thousands of users within New York City. Although, the available data were collected between January 2012 and December 2013, we consider only the user interactions from January 2013 to October 2013.

LBSNs are location-based services that utilize location information with GPS enabled mobile phones or devices and facilitates social networking. They not only provide location information for social networks, additionally provide users a social structure derived from locations that they can share ideas especially for the places, review recommendations, and also creates knowledge of common interests, behavior, and activities from individuals' location-tagged data [38]. However, this data type presents several important limitations. First, the representativeness of social network data may not reflect the characteristics of the population as a whole in terms of socio-economic status, age, gender, or race, and the representativeness may vary geographically. In addition, the problem of representativeness can be extensible to people left behind by the "Digital Divide" who are under-represented [39]. Second, the social network API allows access to a limited number of records [12]. Human mobility time series computed from insufficient samples tend to have more fluctuations, making the general pattern less recognizable and less reliable. Third, unlike the passively collected data from mobile telephone records, smart cards, and wireless networks, social media data have their own intrinsic active nature, as users must grant permission to share their data and determine the location accuracy of their posts, all depending on their personal settings [39]. The LBSNs dataset selected for our work is Gowalla. It is a location-based social networking website where users share their locations by checkingin. The friendship network is undirected and was collected using their public API [40], and consists of 196,591 nodes and 950,327 edges. We have collected a total of 6,442,890 check-ins of these users over the period of February 2009-October 2010. However, this dataset stores a large amount of data and we only consider data from March 2010 to October 2013.

GPS-derived metrics are primarily used for population-level mobility analyses. Location data can, however, also be derived by triangulation from cell towers, from Bluetooth interactions, and from IP addresses via wi-fi connections. GPS data sources can also be complex, with multiple overlapping applications, providers, publishers, and aggregators [41] Prerequisites to data sharing is another limitation. The benefits of using personal data should outweigh the risks to privacy. Baselines need to be established. To compare data with the same time window in previous years seasonal mobility patterns are important; however, most companies or providers do not store data for such a long time because of data retention policies. Clear communication of the baseline is important, including the uncertainty associated with it, so that decision makers can make sense of the changes they see in human mobility. To preserve privacy, data should be aggregated. However, policy makers and researchers will typically request high-resolution disaggregated data. Finally, the GPS dataset was obtained through a Freedom of Information Law (FOIL) request from the New York City Taxi and Limousine Commission (NYCT\&L). The NYCT\&L display trip data of Uber pickups in New York City [42]. This dataset consists of over 4.5 million Uber pickups from April 2012 to September 2013, and 14.3 million more Uber pickups from January to June 2013. For this article, we use the data from January to June 2013.

\subsubsection{Human Mobility Metrics}

Common data-driven human mobility metrics could be useful for observing population mobility. They are generated from CDR, GPS, wi-fi or LBSN by data fusion and analytics. Therefore, the following six metrics are used to comprehensively evaluate people's behaviors. 
- Social-based categorization: a unit of measurement that contributes to data analysis such as unique users or unique devices. It is also related to contacts of people since it considers ICT, recontact rate, contact duration, node density properties;

- Spatial-based categorization: a unit of measurement that categorizes the dimensions of the user locations. For example, tile grids, GPS from POIs, or tower catchment areas define unique locations that are visited by a user for internal analysis;

- Spatial-based aggregation: a unit of measurement that aggregates data of the regions of interest before being shared. It also describes the size of these regions. For example, all users in a region of interest, such as county or neighbourhood, can be aggregated so that the metrics calculate the across population;

- Temporal-based categorization: a unit of measurement that categorises every user's location in specific time periods. For example, the modal location a that a user logs data in every day;

- Temporal-based aggregation: a unit of measurement that aggregates data of all users in a region into a time window. For example, we might be interested in the average numbers of locations visited by users in region over the course of an $8 \mathrm{~h}$ time window;

- Temporal thresholds: a unit of measurement where providers may decide to calculate locations where the user spends at least a certain amount of time, rather than to calculate locations by top location every $8 \mathrm{~h}$;

- Movement-based: a unit of measurement that results from individuals' mobility traces. It considers flight length, pause time, visit frequency, mean-squared distance, number of places visited, or speed;

- Link-based: a unit of measurement that analyzes the similarities or spatio-temporal relations of people movements. Contact duration, node density, intercontact time, recontact rate/count, the temporal variance of node density, pairwise distance, relative velocity are examples of this metric;

- Network performance-based: a unit of measurement that analyzes the effects of human mobility on the performance of the networks. For example, message delay, data loss ratio, transmission count, energy consumption are properties of this networkrelated metric.

Researchers have utilized such data to understand individual human mobility patterns, evaluate human mobility behaviors and explore social ties in several contexts, e.g., on extreme events [43-45].

\subsection{Human Mobility Modeling and Prediction}

The emergence and expansion of smart cities is an excellent example of the use of the IoT and the use of AI, in which ubiquitous computing systems are collecting and generating huge amounts of data daily that not only require a storage location, using Cloud Storage, but also immediate processing, helping citizens take advantage of these data. Decision support systems can also be complemented by analysis of human mobility using DL models, such as trajectory generation (TG), next-location prediction (NLP), and crowd flow prediction (CFP), or mathematical models such as gravity model (GM) and spatial equilibrium model (SEM). Therefore, our work can lead with both techniques using human mobility data network present in Section 2.2.1.

\subsubsection{Challenges and Opportunities}

Based on literature we discuss some challenges such as next-location prediction (NLP), crowd flow prediction (CFP), and trajectory generation (TG). In NLP, individual's mobility patterns, geomarketing and recommendation of friends in social network platforms or location-aware advertisements are some areas that benefit with predicting people's movements [46-48]. However, it also presents many challenges because human mobility is mainly encoded in the temporal order of the visitation patterns and characterized by a high degree of predictability [49]. Furthermore, it can be based on other aspects, such as 
weather conditions and social contacts. Thus, NLP poses the main challenge that is the representation of spatio-temporal characteristics of human movements.

CFP is another important task. Its challenge is to predict in a geographic region the incoming and outgoing flows [50]. It may help mitigate or prevent massive crowds of people streaming into a strip area and other dangerous situations [51,52]. However, CFP presents a problem because it deals with spatio-temporal dependencies. In spatial dependencies, a model should consider that an area's out-flow may affect the in-flows of other areas. Regarding the temporal dependencies, human movements are interpreted by three properties: temporal trends, periodicity, and closeness. Trends are patterns that repeat over time (e.g., working days, months, or years); periodicity consider the repetitive relevant events (e.g., rush hours in the evening); temporal closeness highlights events that are close in time. Moreover, these properties can be affected by external factors such as weather conditions.

The goal of TG models is to generate synthetic trajectories using realistic mobility patterns [53]. The synthetic trajectory model should reproduce a set of spatio-temporal mobility patterns. This model is relevant in many applications. For example, they perform analysis of networking protocols (e.g., mobile ad hoc networks), where network users are exploited to route and deliver the messages [54]. However, TG models capture, simultaneously, the spatio-temporal of human mobility patterns. In its turn, realistic mobility patterns should reproduce the number and sequence of visited locations together with the time, duration of the visits, including the temporal statistics observed empirically. It is challenging to characterize the distance heterogeneity of individuals [55], the tendency to move within short distances [56], daily trajectories of people [57], and the number of locations are constantly visited by individuals in time [58].

Based on previous models, advances in DL models are the most exciting in the AI area. They present evolution in classification and prediction accuracy without the need to use feature engineering (selection, from the raw data, of characteristics that can be used in predictive models) associated with traditional learning supervised. Instead of being programmed, these systems can be trained [59]. However, there are limitations in sight. Processes require large volumes of labeled data to accurately perform complex tasks and obtaining these large datasets can be difficult. Even when they are available, labeling efforts require massive human resources. Furthermore, it can be difficult to know how a mathematical model trained with deep learning arrives at a prediction, recommendation, or decision [60]. Therefore, we present other two emerging solutions for scholars of human mobility prediction: gravity model (GM) and spatial equilibrium model (SEM).

In its turn, GM has demonstrated a great potential for human mobility research. It can be used as a representative model for trip generation and distribution, which are both stages of the several steps model for human flow prediction, where it traditionally used to predict trade flows across countries. Another gravity's advantage lies in its ability to use real data to assess the sensitivity of human mobility flows with respect to policy factors we are interested in. For example, [61] discusses the application potential of the GM in regional science and geography, and in spatial sciences in general (including trade theory) and models international trade flows by means of these models and gravitational gradient models, using Dutch export flows as a test case. Although the GM demonstrated good predictive capacity, affect trade costs, and their impacts on trade flows. It can also be applied in other kinds of applications. For example, the model can clearly explain the spatial dynamics of infectious-disease spread. [62] incorporates trip duration into the mechanism of disease spread, and based on this model, understand how fast and how far a pathogen might spread in a human population.

Finally, SEM is another trend model for human activity analyses. It solves the simultaneous equilibria of plural regions and implies that utility levels are equated across between two regions. [63] indicates that standard spatial equilibrium models generally do a good job in describing the spatial distribution of economic activity and the factor prices of labour and land, but these models also important for urban activity location and travel choices in 
large city regions that anticipate major development or restructuring. For example, [64] consider the spatial relationship between urban green space (UGS) and human activity intensity (HAI) important for urban planning and management, especially for ecological construction of big cities. The spatial equilibrium revealed the spatial correlation between UGS and HAI from the perspective of the ecosystem service (ES), processing and analyzing the whole urban UGS and HAI. In its turn, [65] propose and calibrate a dynamic stochastic general equilibrium (DSGE) model to understand the effect of infectious disease outbreak on tourism. Infectious disease outbreaks, including coronavirus, greatly jeopardize the tourism industry, given its reliance on human mobility.

\subsubsection{Models and Methods}

To solve NLP, some surveys have proposed various DL approaches. For example, based on RNN technique, flashback gives a sparse semantic trajectory, predicts the next point of interest (POI) by looking for similar trajectories in terms of temporal characteristics. It is evaluated using social media posts from [66]. In variational attention based next location (VANext), the use of separate encoders to represent the semantic relationships among POIs provide historical and current trajectories in datasets [67]. DeepMove is an RNN for mobility prediction from sparse trajectories. In addition, it embeds multiple factors that govern human mobility, capturing complicated sequential transitions [68].

CFP implements several DL approaches. It uses local-dilated region-shifting network (LDRSN) that consists of five modules. The first module is convolutional residual unit. It leads local spatial dependencies. The second module uses dilated units. Dilate unit deals with distant spatial dependencies and, being a convolution, captures the correlation of two areas. The outputs of these two modules are forwarded and fused to two other modules. Although one handles short-term temporal patterns, the other has long-term temporal dependencies. The long-term module is followed by a long short-term memory (LSTM) model. This model is evaluated on the dataset of taxis' in New York City using MAPE, RMSE, and MAE [52]. Deep spatio-temporal with irregular convolutional residual network (DST-ICRL) combines LSTMs with convolutional residual units. Similarly to spatial-temporal dynamic network (STDN), DST-ICRL uses three pipelines to uncover different time series and spatio-temporal dynamics. In the experiments, in [69], the authors evaluated the flows from mobile phone data, taxi trajectories data, metro or bus swiping data, and bike-sharing data. Other authors use ConvLSTMs to characterize the spatio-temporal dependencies. For example, they take as input a spatial and temporal sequence composed of the number of bicycles in an area. [70] denotes the spatio-temporal dependency, representing the demand for picking up or returning public bikes. The metrics used for the evaluation are the RMSE and the MAE.

In TG, we can highlight several projects. Being a model-free GAN framework, MoveSim model domain knowledge of human mobility regularity. For example, [71] conducts experiments on two real-life mobility datasets (i.e., mobile operator and GeoLifeGPS), demonstrate that our framework outperforms seven state-of-the-art baselines significantly in terms of improving the quality of simulated mobility data by $35 \%$. This model is evaluated based on a set of attributes, such as the radius of gyration, number of locations visited daily, G-rank represented in a trajectory dataset. [72] presents a generative model called sequential variational autoencoder (SVAE). This model is based on the combination of two models: LSTM and variational autoencoder (VAE). It combines the ability of LSTMs to process sequential data with the ability of VAEs to construct a latent space that captures salient features of the training data. Then, in data processing, the generative model forces the input trajectories to have fixed timestamps. Lastly, the authors evaluate the SVAE through the mean distance error (MDE). In its turn, Yin et al. [73] trains GAN-based framework to generate density distributions using two datasets extracted from MoMo and taxis in San Francisco. The framework was measured using mean absolute error (MAE), mean squared error (MSE), root mean squared error (RMSE), and mean absolute percent error (MAPE). 
The original GM applied in analysis of population included only two variables: population size and spatial distance [74]. Since then, many studies added other variables such as age, politics, income, education, and so on in model. The gradual incorporation of these factors into the model is contributing in explanation of migration phenomena [75-77]. For example, a GM describing the spatial interaction is used to predict migration between cities [78]. It was also used to predict the observed movement patterns to control malaria transmission in Africa. A study concluded that model movement rates between pairs of locations increase with population size and decrease with distance [79]. Other works also seek to understand what are the best parameters to use to obtain the best predictions of human mobility with this model. For example, [80] concluded that appropriate choices of input data and fitted parameters improves the predictive power of spatial interaction models like GM.

According to Samuelson [81], in the financial point of view, SEM adds the consumer's and the producer's surplus to provide a market equilibrium might be obtained by maximizing the net social payoff function (NSP). This model assumes that consumption and production in two different areas connected by a transportation network satisfy based on equilibrium two conditions:

- $\quad$ With $C$, the $P$ is equal to the TC plus the $S P$;

- Without $C$, the $P$ is lower than the TC plus $S P$.

where $C$ is commercialization between pair of markets, $P$ is price, $T C$ is transportation cost and $S P$ is supply price. Inspired model by SEM, Roback analyzes how economic agents locate through space [82]. However, [83] improved this standard SEM. Besides the mobility is determined by preference shocks for locations, such as Moretti [84], he also moved costs that are origin-destination dependent. All these transportation science and spatial economics approaches are fundamental to predict human mobility fluxes between locations.

As we mentioned, the DL algorithms of our work use large volumes of data to learn complex patterns about the movements of people. Their predictive models reveal if the entity is operating within acceptable parameters or is exhibiting an anomaly. Moreover, it requires diverse training data—collected across multiple time frames-to make algorithmic assessments more real-world and accurate. However, to broaden and enrich the correlations made by algorithms, it needs data from diverse open-source datasets.

For the GM and SEM to be estimated, it is also necessary to merge databases from different sources. The large size of the typical database of a gravity model is due to the basic principle that even if the researcher is interested only in the factors influencing a particular trading relationship the effect should be measured based on a gravity equation estimated for all countries, not just the countries involved. In addition, these equations can be estimated for either cross-sections or panels of locations.

Both machine learning and mathematical models researcher should choose the best attributes to apply in models, denying the involvement of the remaining variables of the dataset. Another difficulty related to building a database for these models that data from a variety of different data sources have to be merged in a single database. Since data may be available in different formats or classifications, the researcher needs to organise this information.

\section{Human Mobility Research in Evolution Mobile Network}

The scientific value of observing human mobility has long been applied in a variety of disciplines. This value is growing in parallel with the evolution of mobile network infrastructure. In fact, the ability of a device to communicate with others and its massive adoption by people demonstrated interconnectedness. Moreover, people stopped being merely passive objects where they operate, but rather agents who make choices about where to spend their time and who modify those places through their presence and activities. Therefore, we should highlight the evolution of mobile communication infrastructures in human mobility research. 


\subsection{Human Mobility Research from $1 G$ to $4 G$}

Mobile phone tracking has been the subject of research that has expanded rapidly over the past decades as an array of scientific applications have become technologically and socially feasible. Additionally, it is one that offers some huge benefits over the more traditional methods of people counting, such as overhead cameras, thermal sensors, and laser beams. Whilst smartphone tracking technology is currently applicable in many surveys, it was not always like this. Therefore, Table 1 presents the metrics (represented in Section 2.2.2) used for the human mobility models along with mobile systems growth.

Table 1. Characteristics of the human mobility metrics in Mobile Networks.

\begin{tabular}{ccccccc}
\hline $\begin{array}{c}\text { Mobile } \\
\text { Networks }\end{array}$ & $\begin{array}{c}\text { Movement- } \\
\text { Based }\end{array}$ & $\begin{array}{c}\text { Link- } \\
\text { Based }\end{array}$ & $\begin{array}{c}\text { Network- } \\
\text { Based }\end{array}$ & $\begin{array}{c}\text { Spatial- } \\
\text { Based }\end{array}$ & $\begin{array}{c}\text { Temporal- } \\
\text { Based }\end{array}$ & $\begin{array}{c}\text { Social- } \\
\text { Based }\end{array}$ \\
\hline 1G & & & $\checkmark$ & & & \\
2G & $\checkmark$ & $\checkmark$ & $\checkmark$ & $\checkmark$ & & \\
$3 G$ & $\checkmark$ & $\checkmark$ & $\checkmark$ & $\checkmark$ & $\checkmark$ & $\checkmark$ \\
$4 G$ & $\checkmark$ & $\checkmark$ & $\checkmark$ & $\checkmark$ & $\checkmark$ & $\checkmark$ \\
$5 G$ & $\checkmark$ & $\checkmark$ & $\checkmark$ & $\checkmark$ & $\checkmark$ & $\checkmark$ \\
\hline
\end{tabular}

First generation $(1 \mathrm{G})$ was developed to communicate with the mobile phones through the network of distributed transceivers. Started in 1974 and completed in 1984, its architecture was based on an advance mobile phone service (AMPS) and total access communications system (TACS) technologies. 1G networks only allowed voice calls to be made, suffered from reliability and signal interference issues, and had limited protection against hackers. In addition, they enabled brief conversations that were limited to a few people for professional purposes. These network limitations were managed from a better understanding of human mobility models. Thus various network-related metrics, such as data loss ratio, transmission count, or energy consumption were considered for the management of this mobile network infrastructure. However, there is a need for a more developed system leading $1 \mathrm{G}$ to evolve to $2 \mathrm{G}[3]$.

The next generation of mobile networks, $2 \mathrm{G}$ has driven the general packet radio service (GPRS), similar to the global system for mobile communication (GSM), with a few refinements which gave it higher data speeds, and enhanced data rates for GSM evolution (EGPRS). EGPRS was an evolutionary upgrade and enhancement to 2G and 2.5G GPRS networks through 1990 to 2003. During 2G cellular phones, some significant features were included, like short message service (SMS), picture messages, voice or image messages (MMS), and to improve communication between users, multiple access techniques are used, i.e., time division multiple access (TDMA), code division multiple access (CDMA), and frequency division multiple access (FDMA). In this mobile network, low-level events coming from the network, such as location and cell updates, and calls set-up can be aggregated using triangulation of the position of each mobile equipment. Consequently, these position algorithms make that $2 \mathrm{G}$ gives relevant contributions to determine human mobility patterns based on the several and anonymous sources of information [85].

From 2003 to 2008, 3G provided services with the ability to transfer simultaneously both non-voice data (e.g., exchanging email, downloading information, or instant messaging) and voice. This standard is basically a smooth evolutionary path of $2 \mathrm{G}$ systems. Some of the 3G standards include universal mobile telecommunications system (UMTS) (3GSM), that is by the third generation partnership project (3GPP), high-speed downlink packet access (HSDPA) which provides a linear enhancement path for UMTS-based 3G networks. This improvement allows higher data transfer speeds, and others protocol networks like wideband code division multiple access (W-CDMA), freedom of mobile multimedia access (FOMA), generic access network (GAN/UMA), high-speed uplink packet access (GHSUPA), and time division-synchronous code division multiple access (TD-SCDMA) [86]. This mobile network provides data to survey people's behavior patterns in cyberspace 
and the physical world in terms of human mobility patterns. In addition, spatial-temporal data of $3 \mathrm{G}$ links cyberspace and the physical world and then contribute to investigate their links with urban functional areas. In other words, from $3 \mathrm{G}$ information we are able to investigate how people behave in cyberspace and the physical world, and we further our understanding of people's behaviors by linking them, for example, with social context.

The speed of the $3 \mathrm{G}$ network quickly became insufficient as technology and smartphones evolved. Despite enabling any mobile device to use a wi-fi hotspot and connect, via dongle, to internet access through a computer, the $4 \mathrm{G}$ drastically improved data transfer speeds. Around the year 2010, 4G was split into two categories 4G and 4G LTE (referred to as just LTE). $4 \mathrm{G}$ had even better quality for voice calls as the data transmitted in the pure packet form due to orthogonal frequency division multiplexing or multiple access (OFDM/OFDMA) technique. In this generation, operators collect a ton of spatio-temporal data which enable location analytics to be applied in a wide range of applications, such as in human mobility by identifying how subscribers are moving across a given area (e.g., most visited location), call detail records data from mobile operators, GPS or wi-fi-based data from location-based social networks, third-party mobile apps data such as Twitter, Facebook or Uber, and so on. Besides that, it opens the door to monetize human mobile data by selling location intelligence insights to third parties [87].

\subsection{G Mobile Network to Explore Human Mobility}

$5 \mathrm{G}$ opens a new era in mobile communication technology. This new communication standard combines different flows between terminals and other technologies which access via different wireless technologies at the same time. Although 1G, 2G, 3G, and 4G have played an important role so far, $5 \mathrm{G}$ is able to really connect the entire world without limits. Therefore, this broad and fast digital connection provides a platform for every academy and industry to boost innovation projects in human mobility.

\subsubsection{Components of 5G Physical Infrastructure}

Service-driven 5G network architecture aims to meet diversified mobile service requirements. It broadens cloud access, core networks, transport and support the underlying physical infrastructure using network functions virtualization (NFV) and software-defined networking (SDN). For better support of diversified 5G services the cloud adoption presents as an efficiently and flexibly solution. In addition, it enables the key technologies of E2E network slicing (E2ENS) by the component-based network functions, and on-demand deployment of service anchors. The transport network consists of SDN controllers and underlying forwarding nodes. SDN controllers generate a series of specific data forwarding paths based on network topology and service requirements. This network architecture also manages network resources and implements E2E automatic slicing (E2EAS).

As Figure 5 shows, E2ENS is a key to evolution and support in diversified 5G services. Based on SDN and NFV, physical infrastructure of 5G network architecture consists of sites and three-layer DCs, i.e, Central Office DC (CODC), Local DC (LDC), and Regional DC (RDC). Sites deploy cloud RAN real-time (RT) functions in the form of pico, micro, and macro base stations using multiple modes like 5G, LTE, and wi-fi. However, these functions require real-time and high-performance, computing capability, and inclusion of a specific dedicated hardware platform. In its turn, three layer cloud DC consists of storage and computing resources. The CODC is closest in relative proximity to the base station side. The second layer is the LDC, and the upper layer is the RDC. Each layer of cloud DCs is connected through transportation network infrastructure.

\subsubsection{Open Challenges and Human Mobility Research Directions}

$5 \mathrm{G}$ technology has already been deployed. Currently, we can estimate the revenue opportunities and its value associated with use cases in human mobility research. We rely on a number of key sources supported by a set of properties. 
In the signaling performed within the core network $(\mathrm{CN})$, a property of SDN, should be optimized for handover and re-routing purposes enabling more better support, scalability and performance to human mobility. Moreover, a faster and efficient $\mathrm{CN}$ signaling is only possible using AI techniques like machine learning [88]. Here, by efficiency, we imply that $\mathrm{CN}$ signalling presents an optimized and reduced cost in transmission, processing, and other related metrics.

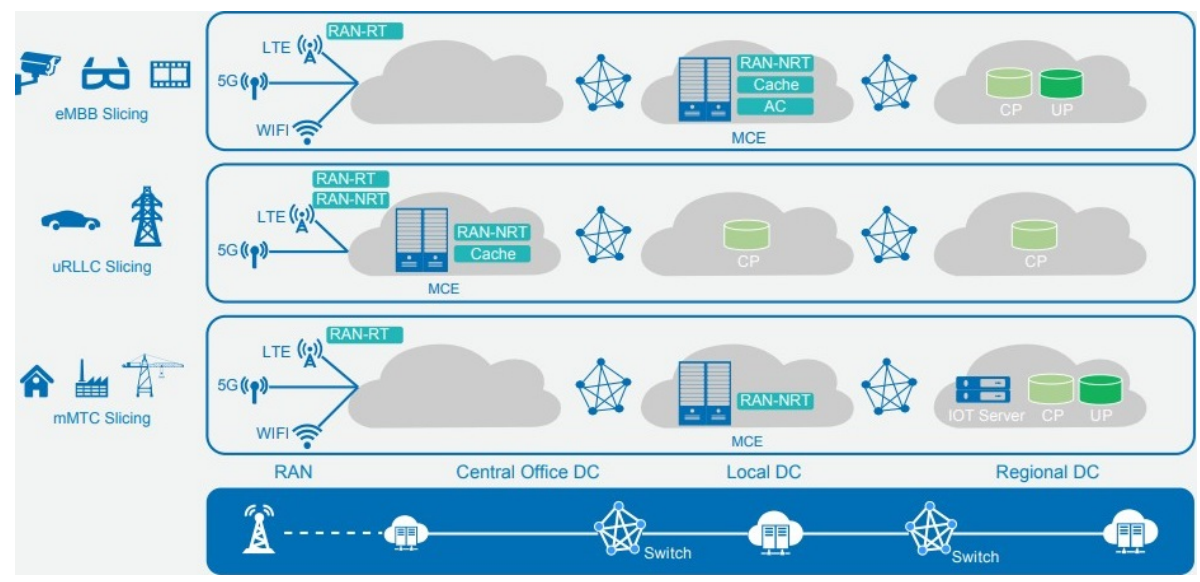

Figure 5. Physical infrastructure of the 5G network architecture (based on [29]).

5G serves different human mobility profiles. Users can effectively access different properties like services or networks with differing loads. For example, to help cater to the different 5G network slices according to their human mobility demands, and avoid the sub-optimal one size fits all approach can be helpful to adopt independent strategies for the various network slices [89]. Moreover, this slice-based strategies enable better network mobility management and quality of service (QoS) provisioning for $5 \mathrm{G}$ networks.

Device-to-device (D2D) works by identifying the human mobility models, individual traces, and features of different human mobility research directions, concerning D2D communication applications in real-life scenarios. For example, 3GPP Release-13 supports communication with devices in clusters, formalizing D2D cluster. Thus, through relaying strategies for $\mathrm{CP} / \mathrm{DP}$ information can enhance the performance of the devices within the network in such clusters. Beyond this extension of CP-DP capabilities of the current D2D framework, policy-based methods also prevail in 5G and B5G networks taking into account the presence of D2D communications and other scenarios between user and device.

User mobility trends or network load are parameters that enable the learning network to plan effective human mobility strategies for $5 \mathrm{G}$ and B5G networks. Furthermore, these dynamic network environments distribute human mobility functionality throughout the network, consequently alleviating congestion issues. Note that network functions virtualization (NFV) and software-defined networking (SDN) facilitate fast switching, while the user-device transits through the network, facilitating network programmability. Furthermore, these service migration strategies of $5 \mathrm{G}$ will greatly enhance the QoS at the same time that users crossover to another public land mobile network (PLMN) and lead to a change in the edge cloud that serves them during human mobility [90].

\section{Emerging Technologies in Human Mobility}

Although neither mobility nor the IoT are new to the business and academic scene, mobile devices are increasingly ubiquitous and IoT has driven an increase in the number of connected devices on the network edge, which creates more traffic across the enterprise network core. To prepare for this increase in traffic, there is a need to invest in connectivity. This higher demand for better connectivity has, in turn, propelled the advancement of emerging network technology. Thus, we should address the impact of human mobility and IoT on mobile networks and outlines how businesses, and academics can prepare those networks for human mobile and IoT growth. 


\subsection{The Impact of Human Mobility and IoT on Mobile Networks}

It is undeniable that the IoT and mobile networks in human mobility research is an emerging research area that encouraging numerous exciting solutions to many problems encountered in numerous domains. However, we should be aware of the several human mobility and IoT challenges and solutions with emerging technologies, devices and applications.

First, the steady increase in mobile device usage means greater demand for bandwidth on existing networks. The challenge here is to design mobile-ready networks that ensure any device in the indoor and outdoor environments has adequate network resources even when connected wirelessly. With hackers becoming even more savvy and the number of vulnerable endpoints growing, device management and securing those endpoints has become a very real challenge. Establishing a security policy that accounts for all devices on a network for every user can be complicated and difficult to enforce. For human mobility research preparing their networks for the future, mobile security should be at the top of the priority list.

Then, IoT has already proven itself a disruptive advancement in technology, transforming the ways people live and work by connecting almost anything from microwaves to doorbells to the internet, gathering data for surveys and making user's lives easier. The problem for human mobility researchers, however, is that the end user's IoT services are created with little regard for enterprise networks, especially when it comes to cyber security. Additionally, to capitalize on the benefits of IoT while maintaining secure and efficient operations, the infrastructures networks should be updated.

Naturally, network performance becomes a main concern in light of all this. Although, typically, data are collected from endpoints and sent to the home server or data center for processing, in IoT this does not work when dealing with vast amounts of data. The volume of human mobility data can be overwhelming and put a great deal of pressure on an network infrastructure. Therefore, the mobile network should be rethought to its foundation in order to improve performance and security while supporting disruptive technologies like IoT.

\subsection{Integration of Emerging IoT Communication Technologies}

As with any emerging technologies, IoT and human mobility bring with them a slew of complications to overcome in terms of the wireless wide area networks (WWAN). To solve it, low power wide area networks (LPWAN) have been a popular low-rate long-range radio communication technology. Sigfox, LoRa, and 3GPP, i.e., extended coverage GSM (ECGSM), long term evolution for machines (LTE-M), and narrowband IoT (NB-IoT), and are the three leading LPWAN technologies that compete for large-scale IoT deployment. Thus, LPWANs and WLANs are fundamentally changing the IoT landscape [91].

Since human mobility environments require devices mobility within similar or heterogeneous LPWAN technologies, supporting mobility refers to ensure the delivery of data on demand and during movement. In other words, the challenges of using IoT in the human mobility research is based on admitting that good network connectivity between devices is essential for communication works well. They must be able to communicate their positions during human movements. Thus, human mobility in this context is defined as the act of moving between gateways (GW) of different technologies and operators. Therefore, it is expected that devices may support more than one technology to achieve timely delivery of human movements information.

In Figure 6, we compare LPWAN technologies and different mobile phone generations by six specific indicators: coverage, spectrum bandwidth, number of base station, power consumption, penetration, and latency. All of them are measured from 0 to 5 ; it can be clearly seen which technology is best in which position. For each indicator, the value 5 is assigned to the technology that has the highest value. The chart has two line series, all coloured differently, one stands for a type of technology; six ticks mean six indicators. 


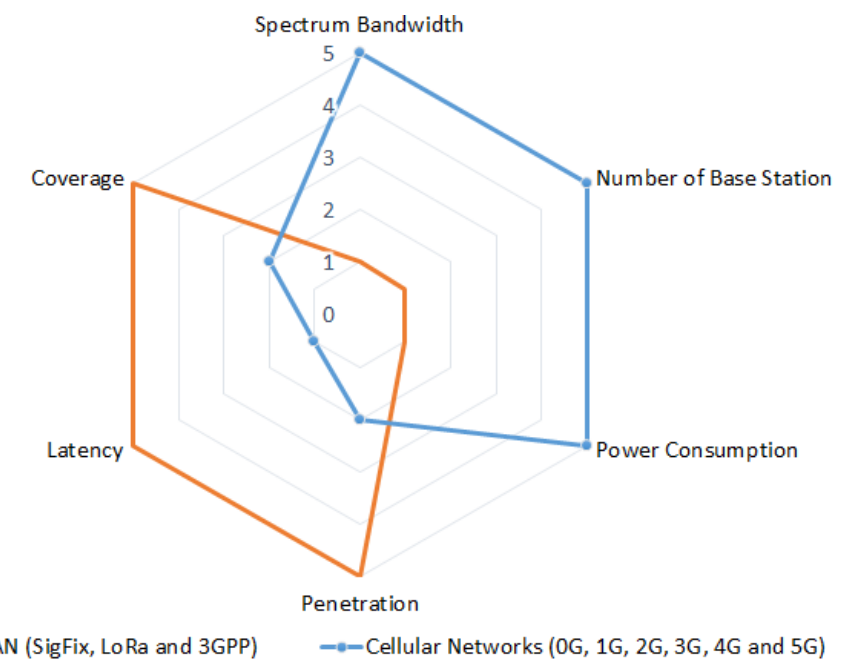

- LPWAN (SigFix, LoRa and 3GPP) - - -Cellular Networks (OG, 1G, 2G, 3G, 4G and 5G)

Figure 6. Qualitative perspective analysis between emerging technologies and traditional cellular.

Although cellular networks require high-band spectrum, installing of the base station and high power consumption, the indicators as coverage, penetration, and latency are the primary drivers for LPWAN applications. However, coverage, energy consumption, cost, and battery life are different characteristics that LPWAN solution may involve significant trade-offs. In addition, they support a plethora of devices in heterogeneous environments with varying expectations. Hence, LPWAN solutions may be customized to cover human mobility applications.

As we mentioned, LoRa, Sigfox, and 3GPP (EC-GSM, LTE-M, and NB-IoT) are the major competitors in the LPWAN space. The end goals of both three technologies are very similar: mobile network operators adopt their technology for IoT deployments over both city and nationwide low power wide-area networks (LPWAN). Although the business models and technologies behind Sigfox, LoRa Alliance, Sigfox, and 3GPP companies are quite different, they use unlicensed communication technologies. In Table 2, we summarize the properties and technical aspects of LoRa, Sigfox, and 3GPP.

LoRa or LoRaWAN networks are an open ecosystem, with strong security specifications, enabling bi-directional communication with focus in capacity, scalability, and optimization for mobility. They present an architecture fault-tolerant and high availability, including a redundant platform. Moreover, LoRa Alliance is creating business efficiencies and improving lives around the world. For example, [92] explores the resource of these networks to monitor fuel tanks used by supply companies. Fill level and other valuable data of these tanks are sent over LoRaWAN networks.

Table 2. Overview of LPWAN technologies: LoRa, Sigfox, and 3GPP.

\begin{tabular}{|c|c|c|c|c|c|}
\hline Parameter & LoRa & SigFox & EC-GSM & LTE-M & NB-IoT \\
\hline Range & $<15 \mathrm{Km}$ & $<50 \mathrm{Km}$ & $<15 \mathrm{Km}$ & $<11 \mathrm{Km}$ & $<15 \mathrm{Km}$ \\
\hline $\begin{array}{l}\text { Max peak } \\
\text { data rate }\end{array}$ & $50 \mathrm{kbps}$ & $100 \mathrm{bps}$ & $74 \mathrm{kbps}$ & $1 \mathrm{Mbps}$ & $250 \mathrm{Kbps}$ \\
\hline $\begin{array}{l}\text { Maximum } \\
\text { coupling loss }\end{array}$ & $157 \mathrm{~dB}$ & $153 \mathrm{~dB}$ & $164 \mathrm{~dB}$ & $160 \mathrm{~dB}$ & $164 \mathrm{~dB}$ \\
\hline Bandwidth & $<500 \mathrm{KHz}$ & $100 \mathrm{KHz}$ & $200 \mathrm{KHz} /$ channel & $1.08 \mathrm{MHz}$ & $180 \mathrm{KHz}$ \\
\hline $\begin{array}{c}\text { Radio } \\
\text { Tecnhology }\end{array}$ & $\begin{array}{c}\text { Spread } \\
\text { Spectrum }\end{array}$ & $\begin{array}{c}\text { Ultra Narrow } \\
\text { Band }\end{array}$ & TDMA/FDMA & OFDM & OFDM \\
\hline Autonomy & $>10$ years & $>10$ years & $>10$ years & $>10$ years & $>10$ years \\
\hline Standardization & $\begin{array}{l}\text { LoRa- } \\
\text { Alliance }\end{array}$ & Sigfox Company & 3GPP & 3GPP & 3GPP \\
\hline
\end{tabular}


Although the Sigfox network business model is differentiated by its top-down approach. Some properties of this network are the cloud server, endpoints software, and the back-end data. Although, the company owns all of these technologies, manufacturers like STMicroelectronics, Atmel, and Texas Instruments have been taking advantage of the fact its an open market for the endpoints and implementation of cost-effective IoT and machine-to-machine (M2M), producing Sigfox radios. However, as the company has exclusive arrangements with network operators, if they work together, only one Sigfox can be deployed in an area [93].

Finally, 3GPP is another emerging technology that defines standards for 5G. Currently, it is made up by different groups, i.e., EC-GSM, LTE-M and NB-IoT which each with a specific focus area. EC-GSM improved security, privacy, and data integrity compared to GSM/EDGE, as well as presents a low cost compared to GSM/GPRS. It can be deployed via software, thereby guaranteeing widespread coverage and accelerated upgrade, benefiting the mobile network features [94]. LTE-M is another component of the 3GPP structure. It reuses existing LTE base stations and supports features such as multicast, higher data rates, positioning, and mobility for inter-frequency measurements [95]. In its turn, NBIoT has a lower cost than LTE-M. Mobility support, reduced bandwidth and data rate, and further protocol optimizations are main simplifications. With the last upgrades, it enhanced positioning for inter-frequency, consumption and latency reduction, improving service [96]. All these groups give a positive contribution to objectives for many works, encouraging new service and application development in human mobility [97-99].

\section{Cellular Networks Technologies}

Based on previous sections, 5G generation of cellular mobile communications revolutionizes communication systems by highly improved network characteristics. It has been designed and optimized for massive spectrum, local offload, advanced coding, and modulation and for utilizing emerge small cell wireless technologies (i.e., LoRa, SigFox, and 3GPP) to improve human mobility coverage. However, this coverage range was not the same in other generations of mobile networks. Thus, in order to better understand the point at which we are at the level of coverage brought by $5 \mathrm{G}$ together with emerging technologies and which allows a wide study of human mobility, we should compare it to previous wireless generations.

\subsection{Coverage Human Mobility Comparison}

The properties of 5G technology improve the QoS, mainly, when large numbers of machines or devices make extensive use of the mobile network. Additionally, these properties provide a good fit for IoT applications. These two benefits can be realized easily in populated areas. Thus, human mobility researchers should focus their research generally only on populated events like music concerts, stadiums, and arenas, and other conventions. For example, in [100], large office towers, such as the Empire State Building, in the USA, or 54-story Mori Tower in Tokyo's Roppongi Hills district, are where thousands of employees work during the week. In many rural households, these 5G deployments improvements do not just extend to phones and tablets-household Internet access via cellular broadband is increasingly common. However, as shown in Figure 7, it was not always so due to the limitations of the coverage range of different mobile networks.

Several human mobility measures indoor or outdoor can be done based on proximity area. Ref. [101] is one such outdoor measure and uses RFID technology. Being RFID an automated identification and data capturing technology that identifies objects without a line of vision and greater reading distance, this work estimates the count of passengers at bus stops, in buses, as well as supply additional buses at required crowded spots. Authors also presents vision-based technique, an alternative for people counting systems. Another idea widely used in human mobility applications to indoor location tracking is also based on RFID. From this technology, ref. [102] proposed an indoor tracking method that enables navigating visually impaired people. It determines a user's location based on the Bayesian 
decision theory considering as an observation vector the signal received by RFID readers. However, the localization error is large because the sensing range of active tags is too far. Therefore, other WPANs technologies that covers a larger area is needed: Bluetooth and Zigbee.

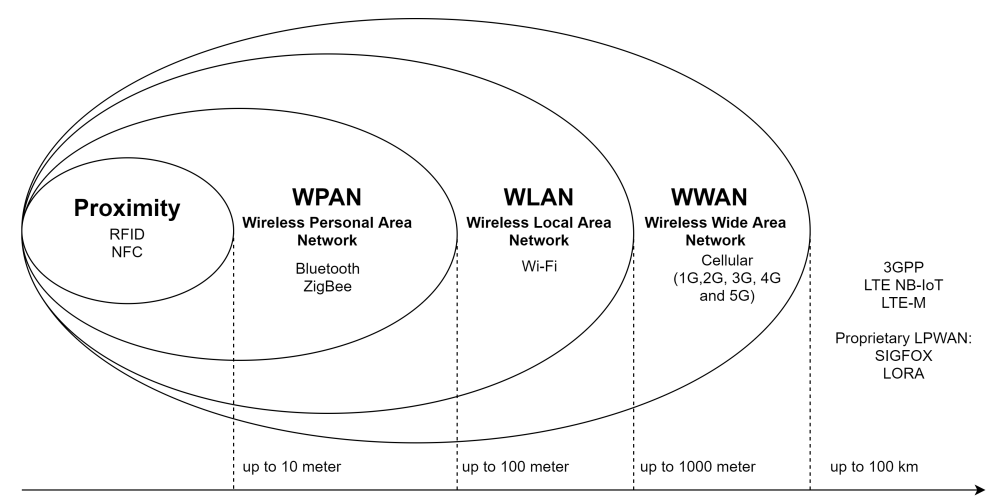

Figure 7. Wireless landscapes.

Bluetooth low energy (BLE) has several benefits such as small size, low cost, and power, and high security. There are several recent research works dedicated to localization systems based on this technology. Beyond exchanges information between devices, BLE provides new opportunities to explore positioning. In [103], researchers study how BLE beacons radio signals can be used for indoor location scenarios, as well as their human mobility tracking precision. In an outdoor context, Zigbee is promising to be of lower cost in comparison with BLE. It is also characterized as low-power consumption, with low data rate, so-called close proximity wireless personal area network (WPAN). In [104], authors identify the existing privacy-preserving solutions, focusing on decentralized methods, and analyzing them in the context of human mobility tracking and proximity detection via mobile device-based localization. One of the drawbacks of using WPAN is operated in a limited area or a personal space around a user. In order to overcome this limitation, human-mobility based surveys can adopt wireless local area network (WLAN).

WLAN has become crucial in indoor positioning. It meets increasing demands for location-based services by ubiquitous connectivity and leveraging the IoT. Moreover, it is essential to determine accurate wireless indoor location information. To deal with all these challenges, for example, ref. [105] uses wi-fi system to data-driven approach that models indoor human mobility at multiple spatial scales, extracting human mobility trajectories from smartphone digital traces. On the other hand, many applications in outdoor environments, such as urban planning, epidemic, and traffic modeling understand human mobility using data collected from WLAN. This communication network enables researchers to found that human mobility can be regular and predictable in outdoor environments [106]. However, it has a limited area to cover and need wireless access points. Then, WLAN by using mobile telecommunication cellular network technologies an even larger area was possible to cover.

1G, 2G, 3G, 4G LTE, and 5G for both indoor and outdoor environments in human mobility researchers were incorporating major requirements, such as inter-operability between various networks, devices and moving bigger data volume resulting from human tracking. They are some of the QoS indicators that are becoming increasingly acceptable. In addition, they could allow for seamless integration of devices and higher speed connectivity on a scale never achieved before. Thus, these mobile networks provide more contributions to implement smart human mobility services where researchers propose cutting edge technologies such as advanced big data analytics (AI/ML). In its turn, emerging technologies (i.e., LoRa, SigFox, and 3GPP) came to further confirm the bet on this type of services. 


\subsection{User Mobility and Data Rates}

All technologies mentioned in Section 5 capture information about user travel at any mobility profile (i.e., fixed or pedestrian). Figure 8 shows that $\mathrm{x}$-axis indicate data rates supported by different cellular access standards and y-axis refers to nature of mobility of the user. Besides, in this visual comparison, we can see that all technologies support high-speed networking. From $1 \mathrm{G}$ to the recent $5 \mathrm{G}$, users in fixed and pedestrian profile offer increased data rates, whereas in wi-fi, better data rates are possible but serve only pedestrian users.

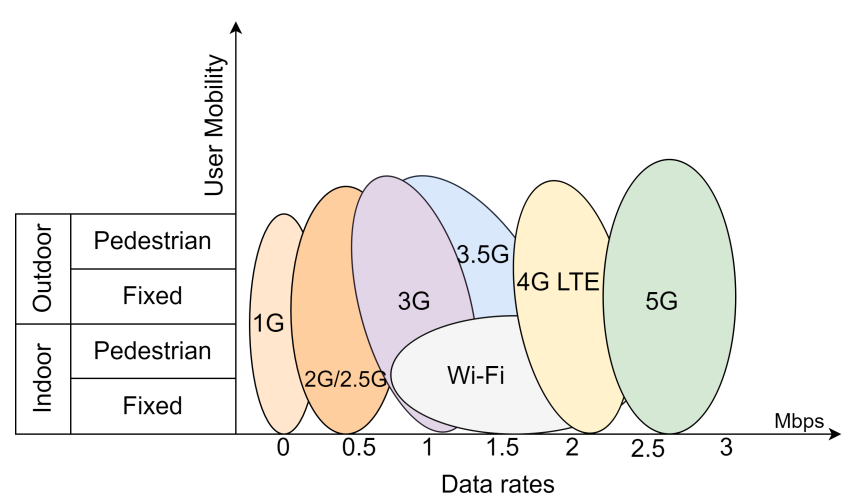

Figure 8. User mobility versus data rates.

Nonetheless, certain challenges in the cellular network technologies and Internet of Things still remain that concern mostly the data rate greater, accessibility, mobility, and reliability. The business aspect of these technologies is flourishing and promising as were the previous generations. In other words, they will be financially beneficial to the economy no matter how successful its technical aspects become. Step by step, users will have to update their devices, or they will have to purchase a device that is compatible with these communication technologies, which produces a high financial turnover for the telecom industry and revives this business every decade. However, the telecom community's experience shows that the success of each new generation will be achieved more slowly on a step-by-step basis than the success of its business aspects.

\section{Conclusions}

The rapidly emerging concepts of mobile network are paving the way to achieve the goal in smart human mobility combining several smart devices, IoT and AI. Initially, this article explores the fundamentals of this idea and provides modern research to monitor and analyze social network topology, spatial distribution, and individual's movements in urban and remote or rural scenarios. Additionally, based on a deep understanding of correlations between these scenarios and spatio-temporal distribution results in a geographical localization, we leverage a set of human mobility data sources and metrics.

Human mobility data and metrics are relevant to a wide array of applications. The results of these applications based on human mobility correlations are expected to inspire strategies to understand and predict human behavior, creating new smart applications and improve existing processes. Furthermore they should include better understandings of information spreading by individuals, improving the network of technological sources of this information like routing protocols and wireless capability. For instance, in making a better prediction of human mobility, we should not only consider the AI model but also the available mobile network that obtains the data. The network dynamics can be the key to collect human interactions with several devices. Therefore, understanding of human movement leads to new information diffusion models across wireless complex networks in the geographical space.

Apart from the huge advantages of IoT, AI, and communications networks, some associated challenges (such as, security, reliability, and big data) and their proposed solutions 
were also presented in this article. Furthermore, this paper has reviewed the exceptional features of $1 \mathrm{G}$ to $5 \mathrm{G}$ cellular network technology emerging with IoT as the next-generation network for supporting the new human mobility requirements. More precisely, detailed information on the role of the 5G cellular network in the progress of IoT and human mobility is delivered. Additionally, some of the problems and solutions associated with this mobile generation were discussed in this study.

Emerging technologies like LoRaWAN, Sigfox, and 3GPP with efficient energy operations, large number of low-cost devices, wide coverage, and a comparatively narrow range of bandwidth were also discussed. These LPWAN solutions are a powerful approach to human traceability. They provide an effective framework and context for carrying out trade-offs for implementations. This paper identifies the gaps and assesses the suitability of LPWAN implementation for human mobility applications. Additionally, it emphasizes a set of solutions like EC-GSM, LTE-M and NB-IoT including developing or updating solutions of these technologies.

We also discuss some of the analysis associated with the human mobility coverage of cellular networks technologies. The upcoming 5G cellular technology is expected to power brand-new applications as its key strength is in the coverage area of user mobility significant improvements in performance and scalability. The future is likely to entail hybrid network solutions with co-existence of personal area networks (PAN), local area network (LAN), wireless wide area networks (WWAN), and LPWANs.

The theoretical contribution of this work brings the most recent scientific and technological bases in the area of human mobility in smart cities. The existing literature shows that various projects (using or not AI) are trying to implement the smart human mobility in cities. These projects have been developed with sophisticated communication technology, using crucial human mobility metrics for implementation in urban areas, as well as the most appropriate AI algorithms. Although, this increasing sciences activities in the human mobility sector has leaded to smart human mobility improvements, they approaches the different technological and scientific components without the perception that there is a set of available technologies in smart cities. Therefore, in this work is harmonized these smart cities and human mobility concepts, so that developing and approaching smart human mobility concept would be easier. Further steps on this research manifests as adjusting these indicators, technologies, and models to most recent methodologies, developing sustainable smart human mobility plans for each city.

Author Contributions: Conceptualization, L.R.; methodology, L.R. and F.S.; validation, L.R., F.S., and C.A.; formal analysis, L.R.; investigation, L.R.; writing-original draft preparation, L.R.; writingreview and editing, F.S. and C.A.; visualization, F.S.; supervision, C.A.; project administration, C.A.; funding acquisition, C.A. All authors have read and agreed to the published version of the manuscript.

Funding: This research received no external funding.

Institutional Review Board Statement: Not applicable.

Informed Consent Statement: Informed consent was obtained from all subjects involved in the study.

Data Availability Statement: No new data were created or analyzed in this study. Data sharing is not applicable to this article.

Acknowledgments: This work has been supported by FCT-Fundação para a Ciência e Tecnologia within the R\&D Units Project Scope: UIDB/00319/2020. This work has also been supported by national funds through FCT-Fundação para a Ciência e Tecnologia through project UIDB/04728/2020.

Conflicts of Interest: The authors declare no conflicts of interest. 


\section{References}

1. Aquilani, B.; Piccarozzi, M.; Abbate, T.; Codini, A. The role of open innovation and value co-creation in the challenging transition from industry 4.0 to society 5.0: Toward a theoretical framework. Sustainability 2020, 12, 8943. [CrossRef]

2. Zikria, Y.B.; Kim, S.W.; Afzal, M.K.; Wang, H.; Rehmani, M.H. 5G mobile services and scenarios: Challenges and solutions. Sustainability 2018, 10, 3626. [CrossRef]

3. Benisha, M.; Prabu, R.T.; Bai, T. Evolution of mobile generation technology. Int. J. Recent Technol. Eng. 2019, 7, $449-454$.

4. Strategy, A. This Report Outlines the Influence of 5G; Technical Report; 2021. Available online: https://www.accenture.com/ _acnmedia/PDF-144/Accenture-5G-WP-EU-Feb26.pdf (accessed on 9 June 2021).

5. Menfors, M.; Fernstedt, F. Geotagging in Social Media-Exploring the Privacy Paradox. Ph.D. Thesis, University of Borås, Borås, Sweden, 2015.

6. Subedi, S.; Pyun, J.Y. A survey of smartphone-based indoor positioning system using RF-based wireless technologies. Sensors 2020, 20, 7230. [CrossRef]

7. Rong, B.; Han, S.; Kadoch, M.; Chen, X.; Jara, A. Integration of 5G Networks and Internet of Things for Future Smart City. Hindawi 2020. [CrossRef]

8. Zheng, Y. Trajectory Data Mining: An Overview. ACM Trans. Intell. Syst. Technol. 2015, 6, 1-41. [CrossRef]

9. Yong, N.; Ni, S.; Shen, S.; Chen, P.; Ji, X. Uncovering stable and occasional human mobility patterns: A case study of the Beijing subway. Phys. A Stat. Mech. Appl. 2018, 492, 28-38. [CrossRef]

10. Wu, R.; Luo, G.; Shao, J.; Tian, L.; Peng, C. Location prediction on trajectory data: A review. Big Data Min. Anal. 2018. [CrossRef]

11. Pamuluri, H.R. Predicting User Mobility Using Deep Learning Methods; Dept. Computer Science \& Engineering, Blekinge Institute of Technology: Karlskrona, Sweden, 2020.

12. Abbasi, M.; Shahraki, A.; Taherkordi, A. Deep Learning for Network Traffic Monitoring and Analysis (NTMA): A Survey. Comput. Commun. 2021. [CrossRef]

13. Ande, R.; Adebisi, B.; Hammoudeh, M.; Saleem, J. Internet of Things: Evolution and technologies from a security perspective. Sustain. Cities Soc. 2020, 54. [CrossRef]

14. Pravir, C.; Gianluca, F.; Stefano, L.; Stefano, L. Mobile Network and BroadBand Coverage Map; JRC103081; European Commission: Ispra, Italy, 2016.

15. Sánchez-Corcuera, R.; Nuñez-Marcos, A.; Sesma-Solance, J.; Bilbao-Jayo, A.; Mulero, R.; Zulaika, U.; Azkune, G.; Almeida, A. Smart cities survey: Technologies, application domains and challenges for the cities of the future. Int. J. Distrib. Sens. Netw. 2019. [CrossRef]

16. Kadar, C.; Pletikosa, I. Mining large-scale human mobility data for long-term crime prediction. EPJ Data Sci. 2018, 7, 1-27. [CrossRef]

17. Hasija, S.; Shen, Z.J.M.; Teo, C.P. Smart city operations: Modeling challenges and opportunities. Manuf. Serv. Oper. Manag. 2020, 22, 203-213. [CrossRef]

18. Zhao, C.; Zeng, A.; Yeung, C.H. Characteristics of human mobility patterns revealed by high-frequency cell-phone position data. Epj Data Sci. 2021, 10, 1-14. [CrossRef]

19. Archer, C.L.; Cervone, G.; Golbazi, M.; Al Fahel, N.; Hultquist, C. Changes in air quality and human mobility in the U.S. during the COVID-19 pandemic. Bull. Atmos. Sci. Technol. 2020. [CrossRef]

20. Wang, A.; Zhang, A.; Chan, E.H.W.; Shi, W.; Zhou, X.; Liu, Z. A Review of Human Mobility Research Based on Big Data and Its Implication for Smart City Development. ISPRS Int. J. Geo-Inf. 2020, 10, 13. [CrossRef]

21. Zhang, D.; Huang, J.; Li, Y.; Zhang, F.; Xu, C.; He, T. Exploring human mobility with multi-source data at extremely large metropolitan scales. In Proceedings of the Annual International Conference on Mobile Computing and Networking, MOBICOM, Maui, HI, USA, 7-11 September 2014; pp. 201-212. [CrossRef]

22. Huang, X.; Li, Z.; Jiang, Y.; Li, X.; Porter, D. Twitter reveals human mobility dynamics during the COVID-19 pandemic. PLoS ONE 2020, 15, e0241957. [CrossRef] [PubMed]

23. Oliveira, T.A.; Oliver, M.; Ramalhinho, H. Challenges for connecting citizens and smart cities: ICT, e-governance and blockchain. Sustainability 2020, 12, 2926. [CrossRef]

24. Camargo, C.Q.; Bright, J.; Hale, S.A. Diagnosing the performance of human mobility models at small spatial scales using volunteered geographical information. R. Soc. Open Sci. 2019, 6. [CrossRef] [PubMed]

25. Khan, M.; Babar, M.; Ahmed, S.H.; Shah, S.C.; Han, K. Smart city designing and planning based on big data analytics. Sustain. Cities Soc. 2017, 35, 271-279. [CrossRef]

26. Porru, S.; Misso, F.E.; Pani, F.E.; Repetto, C. Smart mobility and public transport: Opportunities and challenges in rural and urban areas. J. Traff. Transp. Eng. 2019. [CrossRef]

27. Lousado, J.P.; Antunes, S. Monitoring and support for elderly people using lora communication technologies: Iot concepts and applications. Future Internet 2020, 12, 206. [CrossRef]

28. Ignacio, P.E.; Joel, A.V.; Muñoz, O.; Cesar, C.B.; Javier, S.R.; Darwin, A.Q.; Alejandro, B.F.; Andrés, M.F. Small rural operators techno-economic analysis to bring mobile services to isolated communities: The case of Peru Amazon rainforest. Telecommun. Policy 2020, 44, 102039. [CrossRef]

29. Aernouts, M.; Berkvens, R.; Van Vlaenderen, K.; Weyn, M. Sigfox and LoRaWAN datasets for fingerprint localization in large urban and rural areas. Data 2018, 3, 13. [CrossRef] 
30. Misra, J. The Benefits of IoT for Location Tracking and Personal Security; Bridgera. 2018. Available online: https://bridgera.com/thebenefits-of-iot-for-location-tracking-and-personal-security (accessed on 9 June 2021).

31. Digital Future, S.E. Rural Smart Communities: Boosting Rural Economies through Large-Scale Pilots. 2020. Available online: https: / / digital-strategy.ec.europa.eu/en/news/rural-smart-communities-boosting-rural-economies-through-large-scale-pilots (accessed on 9 June 2021).

32. Ramavhona, T.C.; Mokwena, S. Factors influencing Internet banking adoption in South African rural areas. SA J. Inf. Manag. 2016, 18, 642. [CrossRef]

33. Pestre, G.; Letouzé, E.; Zagheni, E. The ABCDE of Big Data: Assessing Biases in Call-Detail Records for Development Estimates. World Bank Econ. Rev. 2020, 34, S89-S97. [CrossRef]

34. Abba, E.; Aibinu, A.M.; Alhassan, J.K. Development of multiple mobile networks call detailed records and its forensic analysis. Digit. Commun. Netw. 2019, 5, 256-265. [CrossRef]

35. NYC Open Data. 311 Service Requests from 2010 to Present; NYC Open Data. 2021. Available online: https:/ / data.cityofnewyork. us/Social-Services/311-Service-Requests-from-2010-to-Present/erm2-nwe9 (accessed on 9 June 2021).

36. Oliveira, L.; Schneider, D.; De Souza, J.; Shen, W. Mobile Device Detection through WiFi Probe Request Analysis. IEEE Access 2019, 7, 98579-98588. [CrossRef]

37. LinkNYC kiosks. LinkNYC Kiosks: Free Super Fast Wi-Fi and That's Just the Beginning; NYC Department of Information Technology \& Telecommunications. 2021. Available online: https://www.link.nyc/how-to-connect.html (accessed on 9 June 2021).

38. Üsküplü, T.; Terzi, F.; Kartal, H. Discovering Activity Patterns in the City by Social Media Network Data: A Case Study of Istanbul. Appl. Spat. Anal. Policy 2020, 13, 945-958. [CrossRef]

39. Giancarlo Ragozini, M.P.V. Challenges in Social Network Research; Lecture Notes in Social Networks; Springer International Publishing: Cham, Switzerland, 2020; p. 245. [CrossRef]

40. Zitnik, M.; Sosi, R.; Maheshwari, S.; Leskovec, J. SNAP: Network Datasets: Gowalla; Stanford Large Network Dataset Collection. 2014. Available online: https:/ / snap.stanford.edu/data / (accessed on 9 June 2021).

41. Kishore, N.; Kiang, M.V.; Engø-Monsen, K.; Vembar, N.; Schroeder, A.; Balsari, S.; Buckee, C.O. Measuring mobility to monitor travel and physical distancing interventions: A common framework for mobile phone data analysis. Lancet Dig. Health 2020. [CrossRef]

42. Shikun, L. Uber Pickups in New York City; NYC Taxi \& Limousine Commission; 2016. Available online: https://www.kaggle.com/ fivethirtyeight/uber-pickups-in-new-york-city (accessed on 9 June 2021).

43. De Salles Cavedon-Capdeville, F.; Ramos, E.P.; Zamur, A.C.G.; Serraglio, D.A.; Odriozola, I.; de Moura Pallone, L.; Damacena, F.D.L.; Yamamoto, L.; Pinheiro, G.M. Climate Change, Extreme Events and Human Mobility in Latin America: Exploring the Links Through National Laws and Policies. In Climate Change Management; Springer: Berlin/Heidelberg, Germany, 2020; pp. 679-700._34. [CrossRef]

44. Muntean, C.I.; Nardini, F.M.; Noulas, A. Understanding human mobility during events in foursquare. In Proceedings of the CEUR Workshop Proceedings, 7th Italian Information Retrieval Workshop, Venezia, Italy, 30-31 May 2016 ; Volume 1653.

45. Yang, Z.; Gao, W.; Zhao, X.; Hao, C.; Xie, X. Spatiotemporal patterns of population mobility and its determinants in Chinese cities based on travel big data. Sustainability 2020, 12, 4012. [CrossRef]

46. Asgari, F.; Gauthier, V.; Becker, M. A survey on Human Mobility and its applications. arXiv 2013, arXiv:1307.0814.

47. Owuor, I.; Hochmair, H.H. An overview of social media apps and their potential role in geospatial research. ISPRS Int. J. Geo-Inf. 2020, 9, 526. [CrossRef]

48. Cheng, S.; Zhang, B.; Zou, G.; Huang, M.; Zhang, Z. Friend recommendation in social networks based on multi-source information fusion. Int. J. Mach. Learn. Cybern. 2019, 10, 1003-1024. [CrossRef]

49. Mendoza, I.; Rydergren, C.; Tampère, C.M. Discovering regularity in mobility patterns to identify predictable aggregate supply for ridesharing. Transp. Res. Rec. 2018, 2672, 213-223. [CrossRef]

50. Kraemer, M.U.; Sadilek, A.; Zhang, Q.; Marchal, N.A.; Tuli, G.; Cohn, E.L.; Hswen, Y.; Perkins, T.A.; Smith, D.L.; Reiner, R.C.; et al. Mapping global variation in human mobility. Nat. Hum. Behav. 2020, 4, 800-810. [CrossRef]

51. Cecaj, A.; Lippi, M.; Mamei, M.; Zambonelli, F. Sensing and Forecasting Crowd Distribution in Smart Cities: Potentials and Approaches. IoT 2021, 2, 33-49. [CrossRef]

52. Tian, C.; Zhu, X.; Hu, Z.; Ma, J. Deep spatial-temporal networks for crowd flows prediction by dilated convolutions and region-shifting attention mechanism. Appl. Intell. 2020, 50, 3057-3070. [CrossRef]

53. Kang, X.; Liu, L.; Zhao, D.; Ma, H. TraG: A Trajectory Generation Technique for Simulating Urban Crowd Mobility. IEEE Trans. Ind. Inform. 2021, 17, 820-829. [CrossRef]

54. Yang, G.J.; Delgado, R.; Choi, B.W. A practical joint-space trajectory generation method based on convolution in real-time control. Int. J. Adv. Robot. Syst. 2016, 13, 56. [CrossRef]

55. Wang, J.; Dong, L.; Cheng, X.; Yang, W.; Liu, Y. An extended exploration and preferential return model for human mobility simulation at individual and collective levels. Phys. A Stat. Mech. Appl. 2019, 534, 121921. [CrossRef]

56. Scott, P.W. Accounting for time-varying inter-individual differences in trajectories when assessing cross-lagged models. Struct. Equ. Model. 2020. [CrossRef]

57. Paik, H.; Kan, M.J.; Rappoport, N.; Hadley, D.; Sirota, M.; Chen, B.; Manber, U.; Cho, S.B.; Butte, A.J. Tracing diagnosis trajectories over millions of patients reveal an unexpected risk in schizophrenia. Sci. Data 2019, 6, 1-10. [CrossRef] [PubMed] 
58. Park, Y.; Kim, W.; Moon, H. Time-Continuous Real-Time Trajectory Generation for Safe Autonomous Flight of a Quadrotor in Unknown Environment. Appl. Sci. 2021, 11, 3238. [CrossRef]

59. Alzubaidi, L.; Zhang, J.; Humaidi, A.J.; Al-Dujaili, A.; Duan, Y.; Al-Shamma, O.; Santamaría, J.; Fadhel, M.A.; Al-Amidie, M.; Farhan, L. Review of deep learning: Concepts, CNN architectures, challenges, applications, future directions. J. Big Data 2021, 8, 53. [CrossRef]

60. Zohuri, B. Deep Learning Limitations and Flaws. Mod. Approaches Mater. Sci. 2020, 2. [CrossRef]

61. Nijkamp, P.; Ratajczak, W. Gravitational Analysis in Regional Science and Spatial Economics: A Vector Gradient Approach to Trade. Int. Reg. Sci. Rev. 2020. [CrossRef]

62. Giles, J.R.; zu Erbach-Schoenberg, E.; Tatem, A.J.; Gardner, L.; Bjørnstad, O.N.; Metcalf, C.J.; Wesolowski, A. The duration of travel impacts the spatial dynamics of infectious diseases. Proc. Natl. Acad. Sci. USA 2020, 117, 22572-22579. [CrossRef]

63. Van den Bergh, J.C.J.M.; Nijkamp, P.; Rietveld, P. Advances in Spatial Equilibrium Modelling: Retrospect and Prospect; Advances in Spatial Science; Springer: Berlin/Heidelberg, Germany, 1996; pp. 379-385._19. [CrossRef]

64. Zhong, J.; Li, Z.; Sun, Z.; Tian, Y.; Yang, F. The spatial equilibrium analysis of urban green space and human activity in Chengdu, China. J. Clean. Prod. 2020, 259, 120754. [CrossRef]

65. Yang, Y.; Zhang, H.; Chen, X. Coronavirus pandemic and tourism: Dynamic stochastic general equilibrium modeling of infectious disease outbreak. Ann. Tour. Res. 2020, 83, 102913. [CrossRef] [PubMed]

66. Nemes, L.; Kiss, A. Social media sentiment analysis based on COVID-19. J. Inf. Telecommun. 2021, 5, 1-15. [CrossRef]

67. Huang, L.; Ma, Y.; Liu, Y.; He, K. DAN-SNR: A Deep Attentive Network for Social-aware Next Point-of-interest Recommendation. ACM Trans. Internet Technol. 2021, 21. [CrossRef]

68. Feng, J.; Li, Y.; Zhang, C.; Sun, F.; Meng, F.; Guo, A.; Jin, D. DeepMove: Predicting human mobility with attentional recurrent networks. In Proceedings of the Web Conference 2018-Proceedings of the World Wide Web Conference, Lyon, France, 23-27 April 2018; pp. 1459-1468. [CrossRef]

69. Yao, H.; Tang, X.; Wei, H.; Zheng, G.; Li, Z. Modeling Spatial-Temporal Dynamics for Traffic Prediction. arXiv 2019, arxiv:1803.01254.

70. Xiao, G.; Wang, R.; Zhang, C.; Ni, A. Demand prediction for a public bike sharing program based on spatio-temporal graph convolutional networks. Multimed. Tools Appl. 2020,1-19. [CrossRef]

71. Feng, J.; Yang, Z.; Xu, F.; Yu, H.; Wang, M.; Li, Y. Learning to Simulate Human Mobility. In Proceedings of the ACM SIGKDD International Conference on Knowledge Discovery and Data Mining, San Diego, CA, USA, 6-10 July 2020; Volume 20, pp. 3426-3433. [CrossRef]

72. Li, L.; Yan, J.; Wang, H.; Jin, Y. Anomaly Detection of Time Series with Smoothness-Inducing Sequential Variational Auto-Encoder. IEEE Trans. Neural Netw. Learn. Syst. 2021, 32, 1177-1191. [CrossRef] [PubMed]

73. Yin, D.; Yang, Q. GANs Based Density Distribution Privacy-Preservation on Mobility Data. Secur. Commun. Netw. 2018, 2018. [CrossRef]

74. Park, H.J.; Jo, W.S.; Lee, S.H.; Kim, B.J. Generalized gravity model for human migration. New J. Phys. 2018, 20, 093018. [CrossRef]

75. Serlenga, L.; Shin, Y. Gravity models of interprovincial migration flows in Canada with hierarchical multifactor structure. Empir. Econ. 2021, 60, 365-390. [CrossRef]

76. Pitoski, D.; Lampoltshammer, T.J.; Parycek, P. Network analysis of internal migration in Croatia. Comput. Soc. Netw. 2021, 8, 1-17. [CrossRef]

77. Chen, Q.; Yan, J.; Huang, H.; Zhang, X. Correlation of the epidemic spread of COVID-19 and urban population migration in the major cities of Hubei Province, China. Transp. Saf. Environ. 2021, 3, 21-35. [CrossRef]

78. Lin, H. The shrinking of Beijing and the rising of Xiong'an: Optimize population migration in terms of transport service. Discret. Dyn. Nat. Soc. 2020, 2020. [CrossRef]

79. Marshall, J.M.; Wu, S.L.; Sanchez, H.M.; Kiware, S.S.; Ndhlovu, M.; Ouédraogo, A.L.; Touré, M.B.; Sturrock, H.J.; Ghani, A.C.; Ferguson, N.M. Mathematical models of human mobility of relevance to malaria transmission in Africa. Sci. Rep. 2018, 8, 1-12. [CrossRef] [PubMed]

80. Hilton, B.; Sood, A.P.; Evans, T.S. Predictive limitations of spatial interaction models: A non-Gaussian analysis. Sci. Rep. 2020, 10, 1-10. [CrossRef]

81. Enke, S. Equilibrium among Spatially Separated Markets: Solution by Electric Analogue. Econometrica 1951, 19, 40. [CrossRef]

82. Roback, J.; Journal, T.; Dec, N. Wages , Rents , and the Quality of Life Wages, Rents, and the Quality of Life. Quality 2007, 90, 1257-1278.

83. Morten, M.; Oliveira, J. Paving the Way to Development: Costly Migration and Labor Market Integration. NBER Work. Pap. 2016. [CrossRef]

84. Moretti, E. Local Labor Markets. Handb. Labor Econ. 2011, 4 Pt B, 1237-1313. [CrossRef]

85. Tosi, D. Cell phone big data to compute mobility scenarios for future smart cities. Int. J. Data Sci. Anal. 2017, 4, 265-284. [CrossRef]

86. Roddam, S. A Rewind of the Evolution from 1G to 5G; Subex, Shankar Roddam. 2020. Available online: https://www.subex.com/ blog/a-rewind-of-the-evolution-from-1g-to-5g/ (accessed on 9 June 2021).

87. David, F. Building a Spatial Data Monetization Solution. David Fierro, Big Data Tech Lead at Vodafone Business. 2020. Available online: https:/ / carto.com/customer-stories/vodafone-analytics-telecommunications / (accessed on 9 June 2021). 
88. Kaur, J.; Khan, M.A.; Iftikhar, M.; Imran, M.; Emad Ul Haq, Q. Machine Learning Techniques for 5G and beyond. IEEE Access 2021, 9, 23472-23488. [CrossRef]

89. Wen, R.; Feng, G.; Zhou, J.; Qin, S. Mobility management for network slicing based 5G networks. In Proceedings of the International Conference on Communication Technology Proceedings, ICCT, Chongqing, China, 16-19 October 2018; pp. 291-296. [CrossRef]

90. Lin, S.; Lo T.; Mao, R.; Chiu, T.; Lu, L.; Nayak, S. Public Land Mobile Network Selection during International Roaming; Technical Report; Technical Disclosure Commons; 2019. Available online: https: / /www.tdcommons.org / cgi/viewcontent.cgi?article=3634 \&context=dpubs_series (accessed on 9 June 2021).

91. Nikoukar, A.; Raza, S.; Poole, A.; Gunes, M.; Dezfouli, B. Low-power wireless for the internet of things: Standards and applications. IEEE Access 2018. [CrossRef]

92. Ibrahim, D.M. Internet of Things Technology based on LoRaWAN Revolution. In Proceedings of the 2019 10th International Conference on Information and Communication Systems, ICICS 2019, Irbid, Jordan, 11-13 June 2019; pp. 234-237. [CrossRef]

93. GmbH, S.G. Sigfox Monitors Telxius Telecommunication Towers; SigFox: Labège, France, 2018.

94. Kousias, K.; Caso, G.; Alay, O.; Brunstrom, A.; Nardis, L.D.; Benedetto, M.G.D.; Neri, M. Coverage and Deployment Analysis of Narrowband Internet of Things in the Wild. IEEE Commun. Mag. 2020, 58, 39-45. [CrossRef]

95. Dian, F.J.; Vahidnia, R. LTE IoT technology enhancements and case studies. IEEE Consum. Electron. Mag. 2020, 9, 49-56. [CrossRef]

96. Gbadamosi, S.A.; Hancke, G.P.; Abu-Mahfouz, A.M. Building upon NB-IoT networks: A roadmap towards 5G new radio networks. IEEE Access 2020, 8, 188641-188672. [CrossRef]

97. Chen, G.; Viana, A.C.; Fiore, M. Takeaways in Large-scale Human Mobility Data Mining: (Invited Paper). In Proceedings of the IEEE Workshop on Local and Metropolitan Area Networks, Washington, DC, USA, 25-27 June 2018; pp. 55-60. [CrossRef]

98. Faktult, M.N. Towards Passive Tracking and Analyses of Human Mobility at Population Scale Oliver Burkhard; Technical Report; Zürich, Switzerland, 2019. Available online: https://www.geo.uzh.ch/dam/jcr:360a79f4-99f9-4e32-8b4d-5b376f6ba9a2/Diss_Burkhard_ PhD_Thesis_2019.pdf (accessed on 9 June 2021).

99. Lutu, A.; Perino, D.; Bagnulo, M.; Frias-Martinez, E.; Khangosstar, J. A Characterization of the COVID-19 Pandemic Impact on a Mobile Network Operator Traffic. In Proceedings of the ACM SIGCOMM Internet Measurement Conference, IMC. Association for Computing Machinery, Santa Monica, CA, USA, 14-16 November 2020; pp. 19-33. [CrossRef]

100. Karsten, J. 5G Technologies will Power a Greener Future for Cities; Brookings Institution; 2016. Available online: https://www. brookings.edu/blog/techtank/2016/11/30/5g-technologies-will-power-a-greener-future-for-cities/ (accessed on 9 June 2021).

101. Asmael, N.; Waheed, M. Demand estimation of bus as a public transport based on gravity model. In Proceedings of the MATEC Web of Conferences 2017, Sharm el-Shiekh, Egypt, 3-6 October 2017; Volume 162. [CrossRef]

102. Farid, Z.; Nordin, R.; Ismail, M. Recent Advances in Wireless Indoor Localization Techniques and System. J. Comput. Netw. Commun. 2013. [CrossRef]

103. Sindian, S.; Khalil, A.; Samhat, A.E.; Crussière, M.; Hélard, J.F. Resource allocation in high data rate mesh WPAN: A survey paper. Wirel. Pers. Commun. 2014, 74, 909-932. [CrossRef]

104. Shubina, V.; Holcer, S.; Gould, M.; Lohan, E.S. Survey of decentralized solutions with mobile devices for user location tracking, proximity detection, and contact tracing in the covid-19 era. Data 2020, 5, 87. [CrossRef]

105. Trivedi, A.; Silverstein, K.; Strubell, E.; Shenoy, P. WiFiMod: Transformer-based Indoor Human Mobility Modeling using Passive Sensing. arXiv 2021, arxiv:2104.09835

106. Heo, S.; Lim, C.C.; Bell, M.L. Relationships between local green space and human mobility patterns during COVID-19 for Maryland and California, USA. Sustainability 2020, 12, 9401. [CrossRef] 